\title{
Self-antigen-presenting cells expressing diabetes-associated autoantigens exist in both thymus and peripheral lymphoid organs
}

\author{
Alberto Pugliese, ${ }^{1}$ Douglas Brown, ${ }^{1}$ David Garza, ${ }^{1}$ Djanira Murchison, ${ }^{1}$ Markus Zeller, ${ }^{1}$ \\ Maria Redondo, ${ }^{2}$ Juan Diez, ${ }^{1}$ George S. Eisenbarth, ${ }^{2}$ Dhavalkumar D. Patel, ${ }^{3}$ \\ and Camillo Ricordi ${ }^{4}$
}

${ }^{1}$ Immunogenetics Program, Diabetes Research Institute, University of Miami School of Medicine, Miami, Florida, USA ${ }^{2}$ Barbara Davis Center for Childhood Diabetes, University of Colorado Health Sciences Center, Denver, Colorado, USA ${ }^{3}$ Department of Medicine and Immunology, Duke University Medical Center, Durham, North Carolina, USA

${ }^{4}$ Cell Transplant Center, Diabetes Research Institute, University of Miami School of Medicine, Miami, Florida, USA

Address correspondence to: Alberto Pugliese, Immunogenetics Program, Diabetes Research Institute, University of Miami School of Medicine, 1450 NW 10th Avenue, Miami, Florida 33136, USA. Phone: (305) 243-5348; Fax: (305) 243-4404; E-mail: apuglies@med.miami.edu.

Received for publication July 26, 2000, and accepted in revised form January 23, 2001.

Recent reports indicate that genes with tissue-restricted expression, including those encoding the type 1 diabetes autoantigens insulin, glutamic acid decarboxylase (GAD), and the tyrosine-phosphatase-like protein IA-2 (or ICA512), are transcribed in the thymus. The reported modulation of diabetes susceptibility by genetically determined differences in thymic insulin levels and studies in transgenic mice provide correlative and functional evidence that thymic expression of peripheral proteins is crucial for immunological self-tolerance. However, there are no specific data about the existence, tissue distribution, phenotype, and function of those cells that express insulin and other self-antigens in the human thymus. We find that the human thymus harbors specialized cells synthesizing (pro)insulin, GAD, and IA-2, mainly localized in the medulla, and we demonstrate such cells also in peripheral lymphoid organs (spleen and lymph nodes). Phenotypic analysis qualifies these cells as antigen-presenting cells (APCs), including both dendritic cells and macrophages. These cells often appear surrounded by apoptotic lymphocytes, both in thymus and spleen, and may therefore be involved in the deletion of autoreactive lymphocytes. Our findings demonstrate the existence of, and define the tissue distribution and phenotype of, a novel subset of APCs expressing self-antigens in human lymphoid organs that appear to be involved in the regulation of self-tolerance throughout life.

J. Clin. Invest. 107:555-564 (2001).

\section{Introduction}

Immunological self-tolerance can be defined as a physiological state in which the immune system does not react destructively against the organism that harbors it (1). Tolerance to self-molecules is established and maintained through complex mechanisms taking place in both thymus (central tolerance) and peripheral lymphoid organs (peripheral tolerance) (2). Mechanisms of positive and negative selection in the thymus are crucial for the shaping of a self-tolerant T-cell repertoire. Lymphocytes with low to medium affinity to self-peptides are positively selected in the thymic cortex, whereas those with high affinity undergo negative selection (clonal deletion) in the medulla. Negative selection involves those lymphocytes recognizing a self-peptide expressed by a self-HLA molecule on the surface of APCs such as dendritic cells (DCs), macrophages, or thymic epithelial cells, and appears to be mediated by apoptosis (3-8).

Lack or loss of tolerance to self-molecules can result in the development of autoimmune disorders. Type 1, insulin-dependent diabetes mellitus (IDDM), is a
T cell-mediated disorder resulting in the destruction of pancreatic $\beta$ cells (9). Common autoimmune targets in IDDM include molecules expressed in pancreatic islets and neuroendocrine cells, such as insulin, GAD (mostly the $65-\mathrm{kDa}$ isoform), and the tyrosine phosphatase-like protein IA-2 (10-13). Hence, lack or loss of tolerance to these molecules may be crucial for the development of islet autoimmunity and IDDM.

Because proteins with tissue-restricted or peripheral expression are thought to be unavailable for presentation in the thymus, tolerance to such proteins can theoretically be achieved only through peripheral tolerance (14). However, recent evidence suggests that peripheral molecules may also be expressed in the thymus through the autonomous, "ectopic" transcription/translation of the corresponding genes. We reported that insulin, GAD, and IA-2 genes are transcribed in human thymus throughout fetal life and childhood (15). Insulin message in human thymus was also reported by others $(16,17)$; insulin, glucagon, and GAD transcripts were also detected in mouse and rat 
thymus $(18,19)$. Overall, transcripts for several selfmolecules have been detected in thymus, including pancreatic and thyroid hormones, neuroendocrine molecules, and other peripheral proteins $(20,21)$. Thymus transplants in transgenic mice provided functional evidence that thymic expression of self-antigens is crucial for the development of self-tolerance (21).

Little is known about the cells expressing insulin and other self-antigens in human thymus. More insight into the phenotype and function of these cells is necessary to better characterize the relevance of thymic self-antigen expression for self-tolerance and autoimmune diseases. We therefore investigated the existence, abundance, tissue distribution, and phenotype of the thymic cells that express self-antigens. We studied insulin, GAD, and IA-2 because we found their genes transcribed in human thymus (15) and because these molecules are representative of both pancreatic hormones and neuroendocrine molecules and are target autoantigens in a common autoimmune disease.

\section{Methods}

Tissues. We studied thymus, spleen, lymph nodes, pancreas (positive control), and lung (negative control) human specimens. The University of Miami Tissue Bank provided fetal and neonatal tissues. Thymus from children and adolescents undergoing cardiovascular surgery (portions of the thymus must be removed during surgery) were provided by B.F. Haynes (Duke University, Durham, North Carolina, USA). Tissues from adult autoptic cases (pancreas, spleen, lymph nodes, and occasionally thymus) were provided by the Cell Transplant Center, Diabetes Research Institute, University of Miami. Tissues were obtained with the approval of the Institutional Review Boards of the University of Miami and Duke University. Thirty-nine cases were selected on the basis of availability and quality of frozen tissue blocks: 17 cases were fetal and 22 postnatal (age range: 20 weeks of gestation to 63 years old; 23 males and 16 females) from diverse racial groups (16 Caucasians, 13 African-Americans, nine Hispanics; and race undetermined for case 1083). Age, race, and sex are shown in Table 1.

Immunohistochemistry and immunofluorescence. Frozen sections $(5 \mu \mathrm{m})$ were thawed and routinely fixed in $20 \%$ buffered formalin, but other fixatives were used as well (Bouin's solution, acetone, air-drying). For immunohistochemistry, sections were stained using the streptavidin-biotin-peroxidase method and aminoethyl carbazole (AEC, red) as substrate for the horseradish peroxidase enzyme (Zymed Laboratories Inc., South San Francisco, California, USA). For double immunofluorescence, incubation with the primary antibody was followed by incubation with a secondary antibody appropriate for the species and immunoglobulin class of the primary antibody, conjugated with either tetramethyl-rhodamine isothiocyanate (TRITC) or FITC. An Alexa-568-conjugate (Molecular Probes Inc., Eugene, Oregon, USA) was occasionally used instead of
Table 1

Characteristics of tissue donors

\begin{tabular}{|c|c|c|c|c|}
\hline Case & Age & Sex & Race & Tissues \\
\hline \multicolumn{5}{|c|}{ Fetal Cases } \\
\hline 1849 & 20 weeks & $M$ & AA & Thymus \\
\hline 3222 & 21 weeks & $\mathrm{F}$ & AA & Spleen \\
\hline 1278 & 22 weeks & $M$ & $\mathrm{H}$ & Thymus \\
\hline 3446 & 23 weeks & $M$ & AA & Thymus \\
\hline 1819 & 24 weeks & M & $\mathrm{C}$ & Spleen \\
\hline 2892 & 24 weeks & $\mathrm{F}$ & $A A$ & Thymus, Spleen \\
\hline 2045 & 25 weeks & $\mathrm{F}$ & $\mathrm{C}$ & Spleen \\
\hline 2041 & 26 weeks & $\mathrm{F}$ & AA & Spleen \\
\hline 3236 & 26 weeks & $\mathrm{F}$ & AA & Spleen \\
\hline 3223 & 27 weeks & $\mathrm{F}$ & $\mathrm{H}$ & Spleen \\
\hline 3444 & 27 weeks & $\mathrm{F}$ & $\mathrm{H}$ & Thymus, Spleen \\
\hline 2013 & 31 weeks & M & $\mathrm{H}$ & Thymus \\
\hline 2053 & 39 weeks & M & $\mathrm{H}$ & Spleen \\
\hline 2040 & 40 weeks & $\mathrm{F}$ & AA & Thymus, spleen, lymph nod \\
\hline \multicolumn{5}{|c|}{ Postnatal cases } \\
\hline 1829 & 2 days & M & $A A$ & Thymus \\
\hline 1383 & 5 days & M & $A A$ & Spleen \\
\hline 2073 & 5 days & M & $\mathrm{H}$ & Spleen \\
\hline 1263 & 1 month & $\mathrm{F}$ & $\mathrm{H}$ & Thymus \\
\hline 1303 & 6 months & M & C & Thymus \\
\hline 1323 & 2.5 months & $\mathrm{F}$ & C & Spleen \\
\hline 1001 & 6 months & M & $\mathrm{H}$ & Lymph node \\
\hline 1225 & 7 months & M & C & Lymph node \\
\hline 1201 & 18 months & $M$ & $A A$ & Thymus \\
\hline 1119 & 18 years & $M$ & $\mathrm{C}$ & Spleen \\
\hline 1086 & 18 years & $M$ & C & Spleen \\
\hline 1176 & 29 years & M & $\mathrm{C}$ & Spleen \\
\hline 1078 & 29 years & $\mathrm{F}$ & $\mathrm{H}$ & Spleen \\
\hline 1089 & 29 years & M & $A A$ & Spleen \\
\hline 1081 & 30 years & $M$ & $\mathrm{C}$ & Spleen \\
\hline 1130 & 31 years & $\mathrm{F}$ & C & Spleen \\
\hline 1111 & 32 years & $M$ & $A A$ & Thymus \\
\hline 1175 & 40 years & $\mathrm{M}$ & $\mathrm{C}$ & Spleen \\
\hline 1132 & 42 years & $M$ & C & Spleen \\
\hline 1083 & 52 years & $\mathrm{F}$ & ND & Spleen \\
\hline 1115 & 56 years & $M$ & C & Thymus, spleen \\
\hline 1106 & 63 years & $\mathrm{F}$ & $\mathrm{C}$ & Spleen \\
\hline
\end{tabular}

AA, African-American; H, Hispanic; C, Caucasian; ND, not determined.

the TRITC-conjugate (see legend to Figure 2). All direct conjugate primary antibodies were FITC conjugates. Slides were treated with Alexa-488 and anti-fade reagents (Molecular Probes Inc.), mounted with a coverslip, and examined with an inverted fluorescence microscope (Leica DM IRB; Leica, Weitzlar, Germany). The microscope was equipped with two highly selective band pass filters specific for TRITC/Alexa-568 (N3, excitation $546 \pm 12 \mathrm{~nm}$, emission $600 \pm 40 \mathrm{~nm}$ ) or FITC (L5, excitation $480 \pm 40 \mathrm{~nm}$, emission $527 \pm 30 \mathrm{~nm}$ ), plus a tripleband pass filter that allows both TRITC/Alexa-568 and FITC fluorescence to become visible (61000V2; FITC excitation $484.5 \pm 1.5 \mathrm{~nm}$, FITC emission $518.5 \pm 1.5 \mathrm{~nm}$, TRITC excitation $555.1 \pm 1.5$ nm, TRITC emission $602 \pm 1.5 \mathrm{~nm}$; Chroma Technologies, Brattleboro, Vermont, USA). Double-stained cells show both orange and green fluorescence and more often variable gradations of yellow fluorescence resulting from the combination of the orange and green fluorescence when using the tripleband pass filter. Individual marker staining can be also observed and assigned to the same cell using the selective band pass 
filters. Negative controls included omission of the primary antibody, incubation with a control isotype antibody as primary antibody, or staining of lung sections that do not express insulin, GAD, or IA-2.

Primary antibodies. Several antibodies were used against each of the self-antigens studied. Insulin/proinsulin: We used two anti-human proinsulin mouse mAb's with no cross-reactivity to insulin (clones M32337 [Fitzgerald Industries International Inc., Concord, Massachusetts, USA] and 3A1 [Research Diagnostics Inc., Flanders, New Jersey, USA]) and an anti-insulin monoclonal recognizing both insulin and proinsulin (clone K36aC10; Sigma-Aldrich, St. Louis, Missouri, USA). IA-2 (ICA512): we used two rabbit sera raised to the intracellular (serum 8959, aa 601-979) and extracellular portions (serum 9218H, aa 389-576) of the molecule (22). GAD: We used a rabbit antibody recognizing both GAD65 and GAD67 isoforms (lot nos. 19040059 and 18080605; Chemicon International, Temecula, California, USA) and a mouse antibody against GAD65 (clone GAD6; Boehringer Mannheim, Indianapolis, Indiana, USA). AIRE staining was performed using a rabbit serum (no. 845) developed in our laboratory. Staining specificity was validated by blocking with the peptide used to raise the serum. Several FITC conjugates were used to stain for CD markers: CD83 (clone HB15A; Beckman Coulter Inc., Fullerton, California, USA), CD11c (clone 3.9; Calbiochem, San Diego, California, USA), CD40 (clones B-B20 [Research Diagnostics Inc.] MAB89 [Beckman Coulter Inc.]), CD14, CD80, CD86 (clones UCHM-1, BB-1, BU63; Research Diagnostics Inc.), CD8 $\alpha$ (clone T8-FITC; Beckman Coulter Inc.), HLA-Class II (clones HK14 [Sigma-Aldrich] and B8.12.2 [Beckman Coulter Inc.]), CD20 and CD19 (Beckton-Dickinson), cytokeratin (clone AE3; Zymed Laboratories Inc.).

Apoptosis detection. Apoptosis was assessed on tissue sections using the terminal deoxynucleotidyl transferase [TdT] dUTP nick-end labeling (TUNEL) assay to detect DNA fragmentation. A FITC-based TUNEL
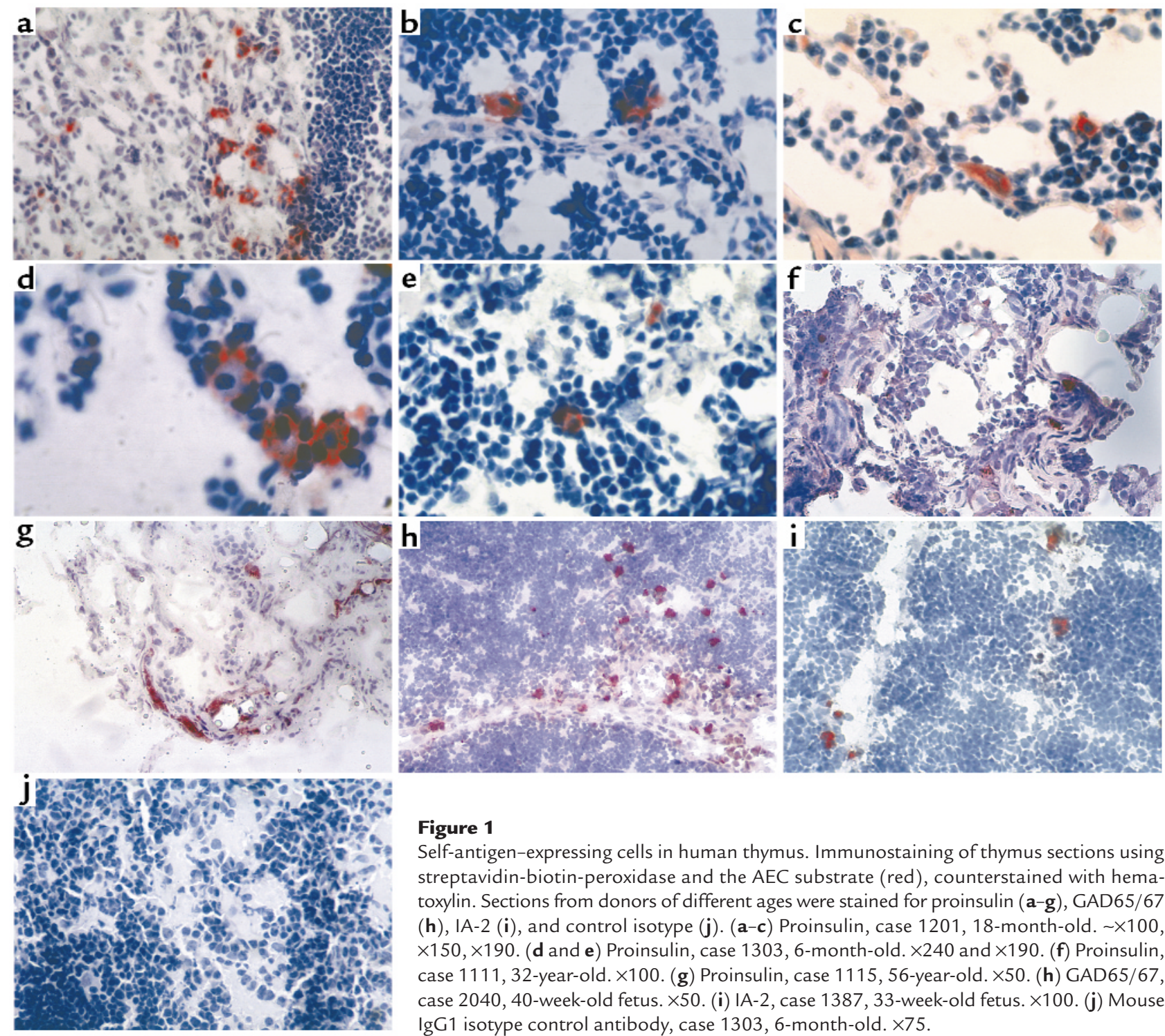

Figure 1

Self-antigen-expressing cells in human thymus. Immunostaining of thymus sections using streptavidin-biotin-peroxidase and the AEC substrate (red), counterstained with hematoxylin. Sections from donors of different ages were stained for proinsulin (a-g), GAD65/67 (h), IA-2 (i), and control isotype (j). (a-c) Proinsulin, case 1201, 18-month-old. $>100$, $\times 150, \times 190$. (d and e) Proinsulin, case 1303, 6-month-old. $\times 240$ and $\times 190$. (f) Proinsulin, case 1111, 32-year-old. $\times 100$. (g) Proinsulin, case 1115, 56-year-old. ×50. (h) GAD65/67, case 2040, 40-week-old fetus. $\times 50$. (i) IA-2, case 1387, 33-week-old fetus. $\times 100$. (j) Mouse IgG1 isotype control antibody, case 1303, 6-month-old. $\times 75$. 
Figure 2

Self-antigen-expressing cell phenotype in thymus. Frozen thymus sections were subject to double immunofluorescence. Left column: double-stained cells exhibiting both TRITC (or Alexa-568) and FITC fluorescence often seen as variable gradations of yellow fluorescence with the tripleband filter. Center and right columns: staining for either one of the two individual markers used, as indicated above each image, using highly selective band pass filters for either TRITC (or Alexa-568) or FITC. Top to bottom: Proinsulin-CD83, case $1897 . \times 84$. Not all cells are double stained. Proinsulin-CD11c, case 3446. $\times 140$. Proinsulin-CD14, case 1829. $\times 90$. Proinsulin-CD $8 \alpha$, case 3446. $\times 90$. Proinsulin-AIRE, case 3446. $\times 90$. The AIRE staining was specifically blocked with the peptide used to raise the rabbit serum. Color differences in the images showing the proinsulin staining (center panels) are due to the use of different conjugates (TRITC for the CD83 and CD14 series and Alexa-568 for the CD11c, CD8 $\alpha$, and AIRE series).
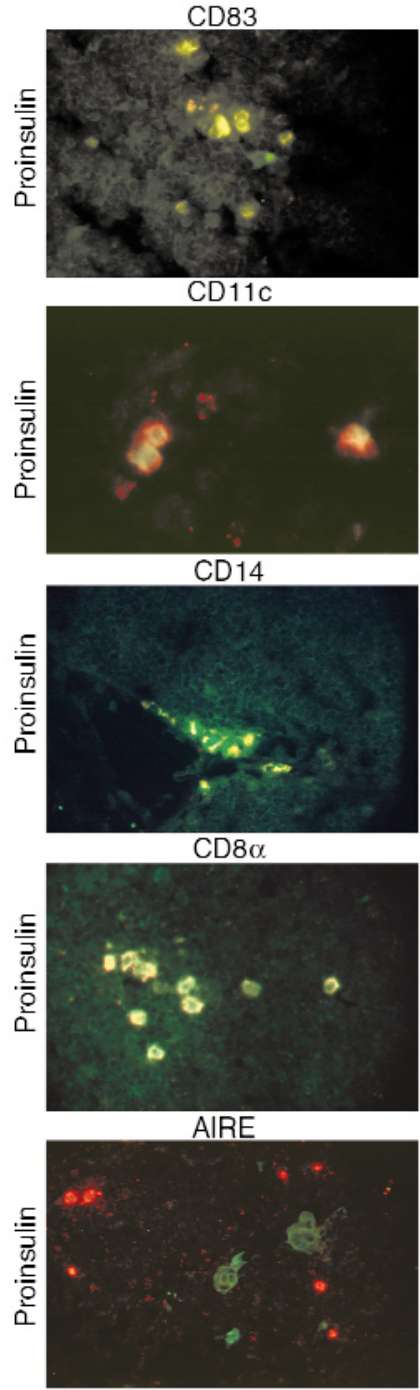

Proinsulin

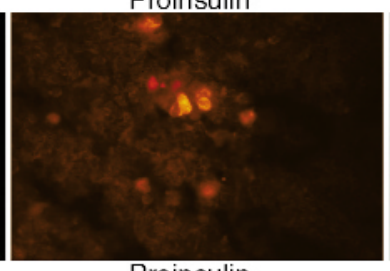

Proinsulin

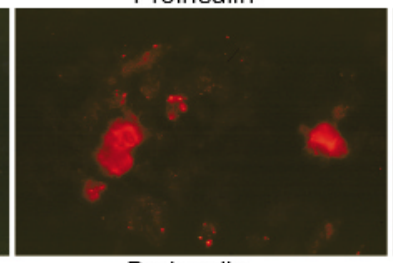

Proinsulin

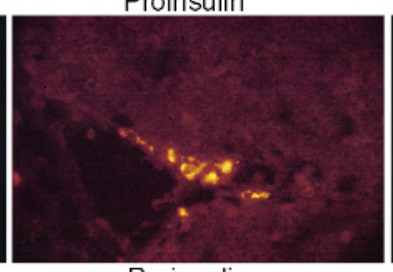

Proinsulin

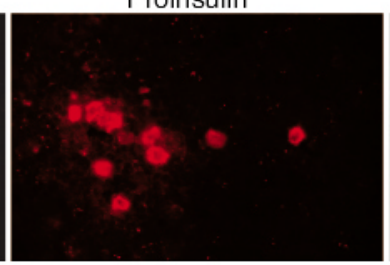

Proinsulin

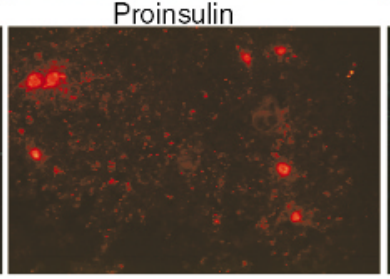

CD83

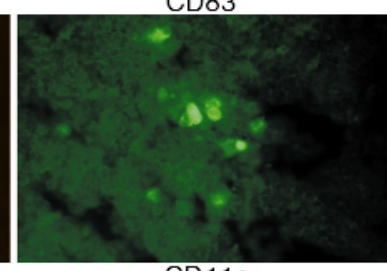

CD11C

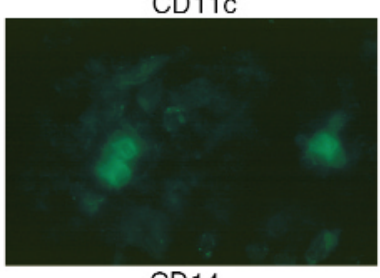

CD14

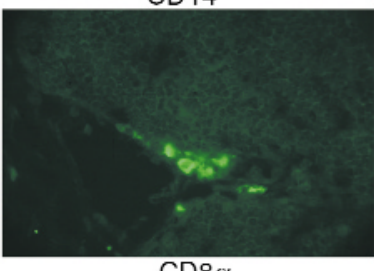

$\operatorname{CD} 8 \alpha$

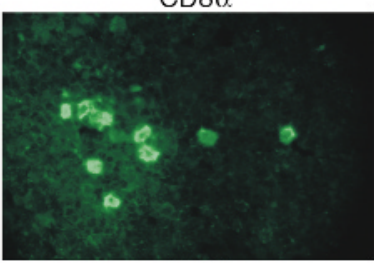

AIRE

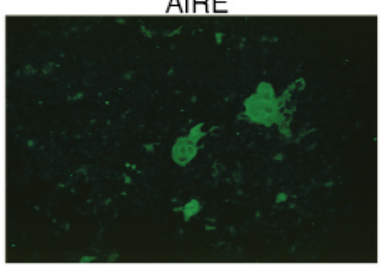

(Promega Corp., Madison, Wisconsin, USA) was combined with proinsulin staining detected with a secondary TRITC-conjugate. A peroxidase-based TUNEL assay (TdT-Fragel; AEC substrate; Oncogene Research Products, Boston, Massachusetts, USA) was coupled with proinsulin staining detected with alkaline phosphatase and AP-Orange as substrate (Zymed Laboratories Inc.). Endogenous alkaline phosphatase activity was inhibited adding $1 \mathrm{mM}$ levamisole to the substrate solution. Controls for the TUNEL staining included sections treated with DNAse (positive control) and omission of the TdT enzyme (negative control).

$m R N A$ expression studies. The expression of specific mRNAs for insulin, GAD, and IA-2 was assessed as described previously (15). After total RNA isolation cDNA was reverse transcribed and then subject to PCR amplification (RT-PCR). All reactions were run for 35 cycles, and products were visualized on ethidium-bromide stained $2 \%$ agarose gels. Primers for the insulin mRNA (437 bp) were GAA GAG GCC ATC AAG CAG ATC ACT G (forward) and GTT CAA GGG CTT TAT TCC ATC TCT C (reverse). These primers do not amplify genom- ic DNA, as the forward primer spans across the first intron. The same technique was used to amplify mRNA for GAD65, GAD67, and IA-2, and $\beta$-actin (15). Genomic DNA or PCR contamination was excluded by running reactions without $\mathrm{RT}$ or template.

Sequencing of PCR products. PCR products were cloned (TA Cloning Vector; Invitrogen Corp., San Diego, California, USA) and sequenced (both strands) with an automated sequencer.

\section{Results}

Self-antigen-expressing cells exist in the buman thymus. Frozen thymus sections were stained using several antibodies against proinsulin/insulin, GAD (65- and $67-\mathrm{kDa}$ isoforms), and IA-2. Figure $1, \mathrm{a}-\mathrm{g}$, shows sparse cells staining positive for proinsulin (two of the three antibodies used were specific for proinsulin, and the third antibody could recognize both insulin and proinsulin). The sections shown are representative of several cases studied ranging in age from 20 weeks of gestation to 56 years (Table 1 ). The eldest specimens evaluated were from 32- and 56-year-old subjects and also con- 
tained proinsulin-positive cells (Figure 1, f and g). This observation is consistent with the demonstration that the thymus is still active in adults, although producing fewer $T$ cells (23). No staining was observed in lung sections, omitting the primary antibody in both thymus and pancreatic sections (data not shown), or using an isotype-matched control antibody (Figure 1j). Pancreas sections were intensely stained in a typical pattern (data not shown). Occasionally, self-antigen-expressing cells would concentrate in a small area (Figure 1a), whereas in most cases, they would distribute sparsely within the medulla or at the corticomedullary junction. Rosettes consisting of thymocytes surrounding proinsulin-positive cells were frequently seen (Figure 1, b, d, and e). Cells with similar appearance, abundance, and distribution were stained with antibodies to GAD65/67 and IA-2 (Figure 1, h and i).

Phenotypic analysis of self-antigen-expressing cells in human thymus. We used double-immunofluorescence to directly colocalize the expression of proinsulin, GAD, and IA2 with known markers that would help define the phenotype of self-antigen-expressing cells. Thymic cells expressing proinsulin, GAD, and IA-2 also expressed CD83, CD11c, CD40, CD14, CD80, CD86, CD8 $\alpha$, and
HLA-class II. Figure 2 shows colocalization of proinsulin with CD83, CD11c, and CD14. Among APCs, DCs express CD83, CD11c, and CD40, whereas macrophages express CD14. We were unable to colocalize any of the islet antigens studied with cytokeratin using the AE3 antibody (data not shown), a marker of epithelial cells. Thus, thymic epithelial cells do not appear to express the islet cell antigens studied. Moreover, we were unable to observe colocalization of proinsulin with the autoimmune regulator protein (AIRE) (Figure 2), a recently discovered transcription factor that is thought to function as an "autoimmune regulator" and is reportedly expressed in thymic epithelial cells and a DC subset (24). In addition, AIRE-positive cells appear larger and morphologically distinct from self-antigen-expressing cells (24).

Self-antigen-expressing cells exist in peripheral lymphoid organs. In contrast to thymic epithelial cells, DCs and macrophages are also present in peripheral lymphoid organs. Because thymic cells expressing self-antigens comprise both DCs and macrophages, we hypothesized that similar cells may exist in peripheral lymphoid organs. We first investigated whether mRNA for insulin and other autoantigens could be detected in spleen and

\section{Figure 3}

Self-antigen-expressing cells in peripheral lymphoid organs. Top panel: expression of insulin mRNA in peripheral lymphoid organs by RTPCR. From left to right: no template reaction (lane 1), thymus 1278 (lane 2, positive control), spleen 1819 (lane 3), lymph node 2040 (lane 4), lymph node 1001 (lane 5), lymph node 1225 (lane 6), spleen 2073 (lane 7), and spleen 2053 (lane 8). The intensity of $\beta$-actin bands differs among samples, suggesting that significant RNA degradation had occurred for some specimens, a phenomenon that cannot always be prevented using human tissues. Bottom panel: immunostaining of spleen (left column) and lymph node (right column) sections using the streptavidin-biotin-peroxidase method and the AEC substrate (red), counterstained with hematoxylin. Sections are stained for proinsulin, GAD65/67, or IA-2, top to bottom. (a) Proinsulin staining, case 1176,29 -year-old. $\times 24$. Note the distribution of proinsulin-positive cells around the white pulp. (b) Proinsulin staining, case 1106, 63year-old. $\times 150$. (c) GAD65/67 staining, case 2040, 40-week-old fetus. $\times 250$. (d) GAD65/67 staining, case 2040, 40-week-old fetus. $\times 100$. (e) IA-2 staining, case 2053, 39week-old fetus. $\times 100$. (f) IA-2 staining, case 2053, 39-week-old fetus. $\times 150$.

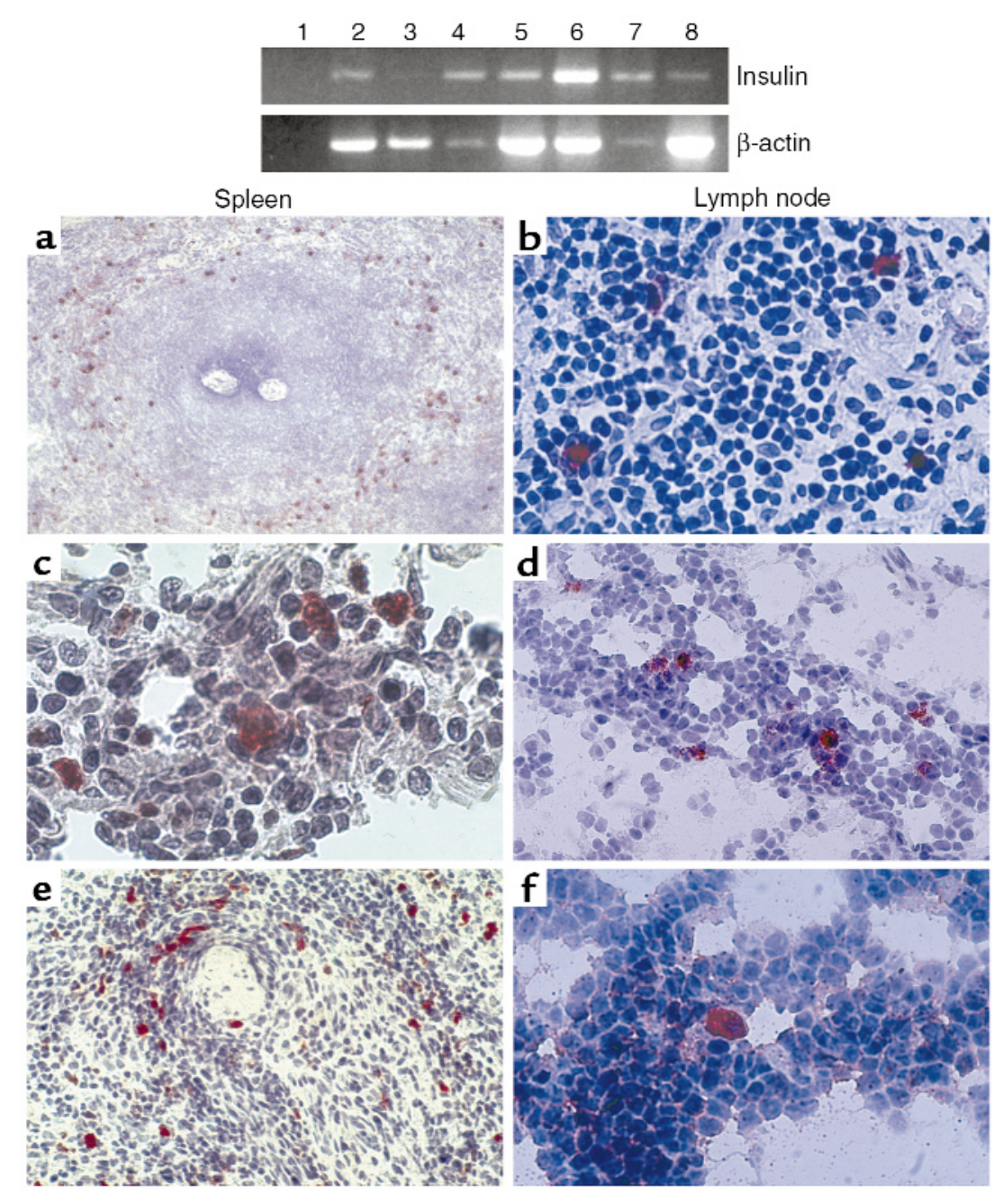




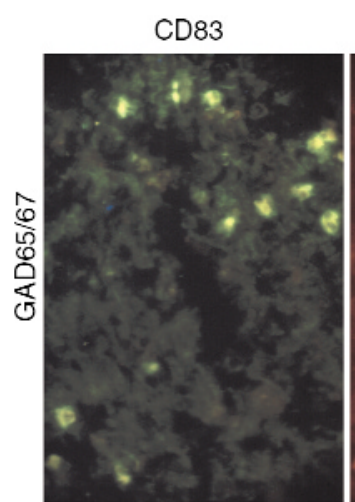

CD40

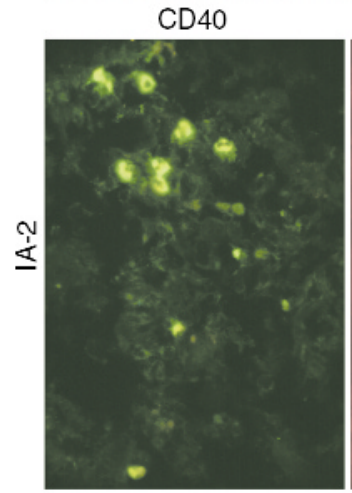

Class II
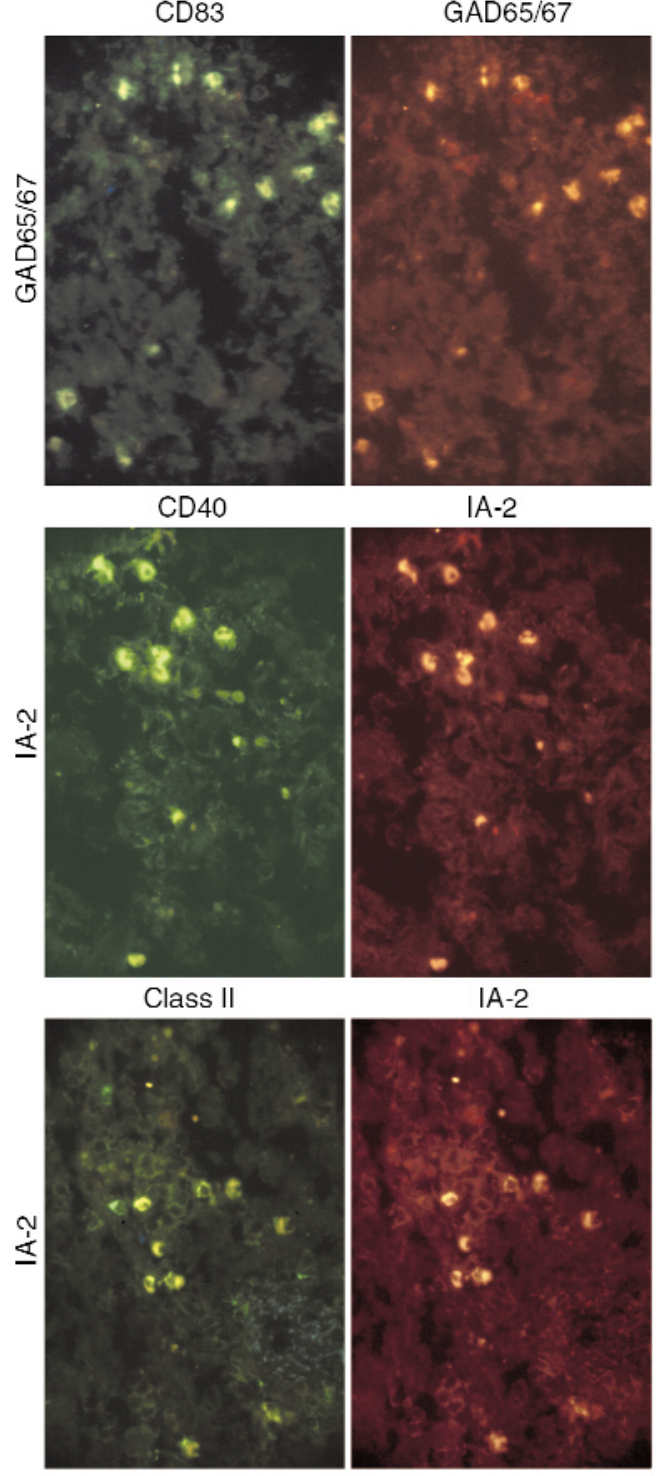

IA-2

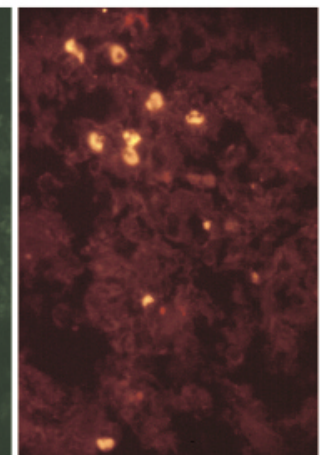

IA-2
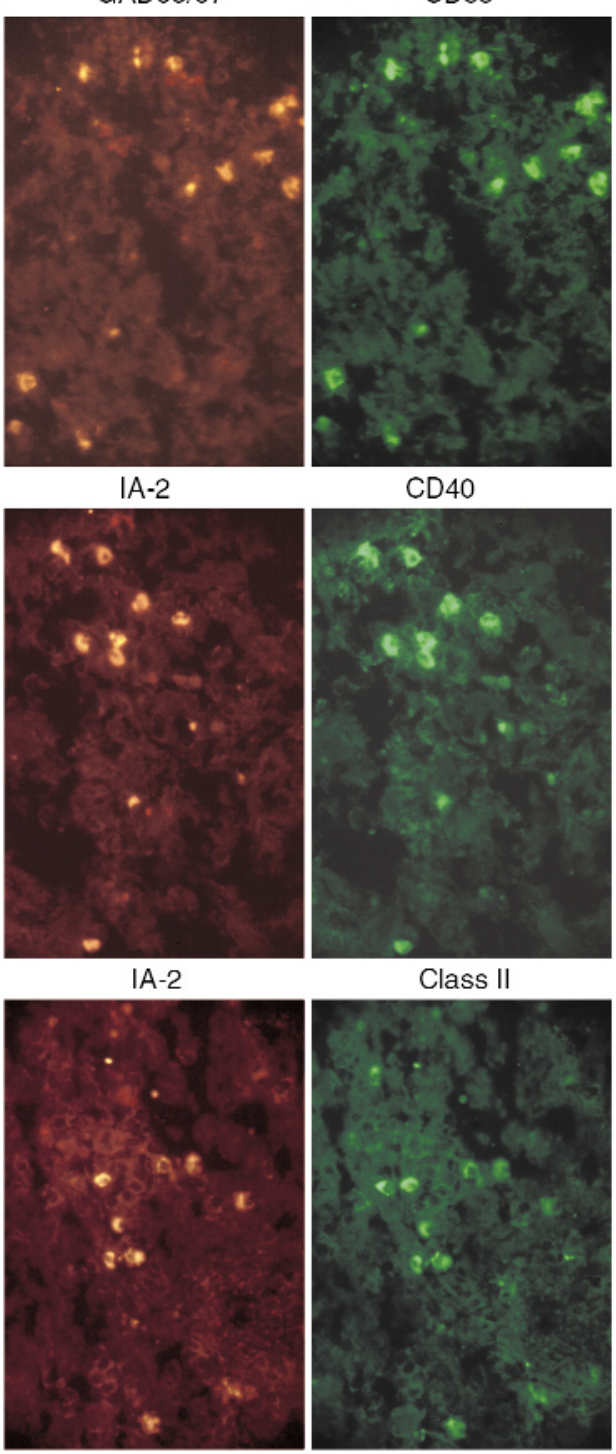

CD40

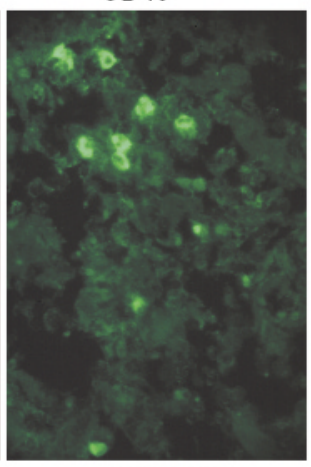

Class II

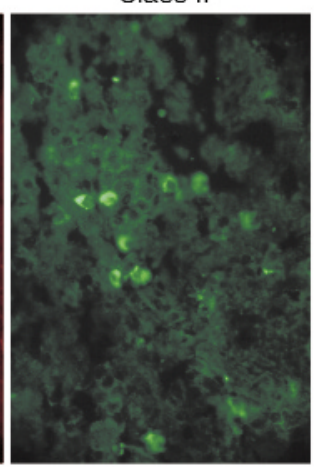

Figure 4

Self-antigen-expressing cell phenotypes in spleen. Top to bottom: double-staining for GAD65/67-CD83, IA-2-CD40, IA-2-HLA-class II (case 1089; ×100; not all cells are double-stained).

lymph node specimens by RT-PCR. We detected insulin mRNA in 16 of 24 and three of six spleen and lymph node specimens tested (Figure 3), as well as GAD and IA2 transcripts (data not shown). Cells expressing proinsulin, GAD, and IA-2 were also detected in both spleen and lymph nodes by immunohistochemistry (Figure 3). The morphology and the sparse distribution observed in the thymus were also noted in peripheral lymphoid organs. Self-antigen-expressing cells were observed in red pulp areas, often surrounding the white pulp areas (Figure 3a). Both the distribution pattern and the lack of colocalization with CD19 and CD20 (data not shown) suggest that self-antigen-expressing cells do not comprise B-lymphocytes. Self-antigen-expressing cells were often found in the center of rosettes also in peripheral lymphoid organs (Figure 3, b and c).
Phenotypic analysis of self-antigen-expressing cells in peripheral lymphoid organs. We investigated whether self-antigen-expressing cells in peripheral lymphoid organs correspond to those in thymus using double-immunofluorescence to analyze the expression of proinsulin, GAD, and IA-2 and several phenotypic markers. Self-antigen-expressing cells from spleen expressed the same phenotypic markers expressed by thymic selfantigen-expressing cells including CD83, CD11c, CD40, CD80, CD86, CD14, CD8 $\alpha$, and HLA-class II (Figure 4). Thus, self-antigen-expressing cells belong to the DC and macrophage lineages both in thymus and peripheral lymphoid organs.

Self-antigen-expressing cells can express multiple self-antigens. We used double-immunofluorescence to directly assess whether self-antigen-expressing cells can express multiple selfantigens. Thymus and spleen sections were simultaneously stained for proinsulin and GAD, IA-2 and GAD, or proinsulin and IA-2 using primary antibodies from different species. The majority of self-antigen-expressing cells were found to be positive for the two antigens for which they were stained (Figure 5), suggesting that self-antigen-expressing cells can express multiple self-antigens. Although it is difficult to estimate the overall abundance of self-antigen-expressing cells by immunostaining, and we noted interspecimen variation, it is possible that $1-3 \%$ of thymic cells may express islet cell antigens. These cells appear more numerous in the spleen than in thymus.

Self-antigen-expressing cells and apoptosis. The predominant localization within the thymic medulla, the formation of rosettes, and the functional studies reported in the mouse (21) strongly suggest that self-antigen-expressing cells may participate in thymic negative selection and mediate tolerogenic signals, some of which may culminate in the apoptotic death of autoreactive lymphocytes. To investigate this possibility, we performed double-staining experiments in which frozen thymus and spleen sections were stained for proinsulin and for the TUNEL reaction, a marker of apoptosis. Proinsulinpositive cells were demonstrated in the center of rosettes in which the surrounding cells were clearly apoptotic, both in thymus and spleen (Figure 6). Although ischemia may contribute to some of the apoptosis observed in autoptic tissues (Figure 6c), apoptotic cells are often seen in intimate contact with the proinsulin-positive cell in thymus (Figure 6, a and b) and spleen (Figure 6, c and d), suggesting that apoptosis in those particular cells may be related to the interaction with the self-antigen-expressing cell. 


\section{Discussion}

We present evidence that the human thymus contains APCs expressing self-antigens known to have tissuerestricted or peripheral expression. We also demonstrate the existence of these cells in peripheral lymphoid organs such as spleen and lymph nodes. These findings and the demonstration that the genes coding for the self-molecules studied here are transcribed in both thymus (15) and peripheral lymphoid organs support the notion that these self-molecules are directly produced and not captured by self-antigen-expressing cells. Both their predominant localization within the thymic medulla and the formation of rosettes are typical of DCs and to a lesser extent of macrophages (7). Our phenotypic analysis shows that self-antigen-expressing cells express typical markers of APCs such as DCs and macrophages, including CD83, CD11c, CD14, CD40, CD80, CD86, CD8 $\alpha$, and HLA-class II, and therefore could be better termed self-antigen-presenting cells. The majority, but not all, of the cells expressing markers of APCs (CD83, CD11c, CD14, CD40, etc.) expressed proinsulin, GAD, or IA-2. Figure 2 shows a CD83-positive cell that does not express proinsulin, Figure 4 (bottom panels) shows a cell that expresses HLA-class II but not IA-2. Many of the cells that did not stain for the self-antigens studied expressed those markers that are not exclusively expressed by APCs, such as HLA-class II and $C D 8 \alpha$. Our findings are in agreement with studies reporting cells expressing insulin and other pancreatic molecules in the mouse thymus that had typical features of DCs and macrophages and were termed peripheral antigen-expressing cells $(21,25$, 26). However, we did not observe the exclusive expression of insulin in DCs reported in the mouse thymus (26).

The rosettes observed in thymus and peripheral lymphoid organs appear to consist of lymphocytes interdigitating with a DC and may represent the microenvironment where self-reactive lymphocytes are deleted (7). Considering that negative selection is classically compartmentalized in the thymic medulla and is mostly mediated by DCs (2), our findings showing expression of CD83 and CD11c suggest that self-antigen-presenting cells include a population of DCs that may be involved in the clonal deletion of self-reactive lymphocytes. The observation, in both thymus and spleen, of rosettes consisting of proinsulin-positive cells surrounded and in intimate contact with apoptotic cells provides morphological, albeit circumstantial, evidence that self-antigen-presenting cells may induce apoptosis of autoreactive $\mathrm{T}$ cells. This is consistent with reports that regulatory DCs kill T-cells by inducing apoptosis (3) and that T-cell apoptosis also takes place in the spleen $(4,27,28)$.

In addition to myeloid DCs, known to be potent stimulators of the immune response, studies in mice suggest the existence of a subset of lymphoid DCs with tolerogenic function (8). The self-antigen-expressing DCs reported here expressed markers of mature myeloid DCs (CD83, CD11c) and CD8 $\alpha$, a marker of lymphoid DCs in the mouse. This is consistent with the postulated tolerogenic function and lymphoid tissue distribution, although the association of CD8 $\alpha$ with an exclusively tolerogenic phenotype is not reported by all studies (8). In the mouse, $C D 8 \alpha$-positive and -negative
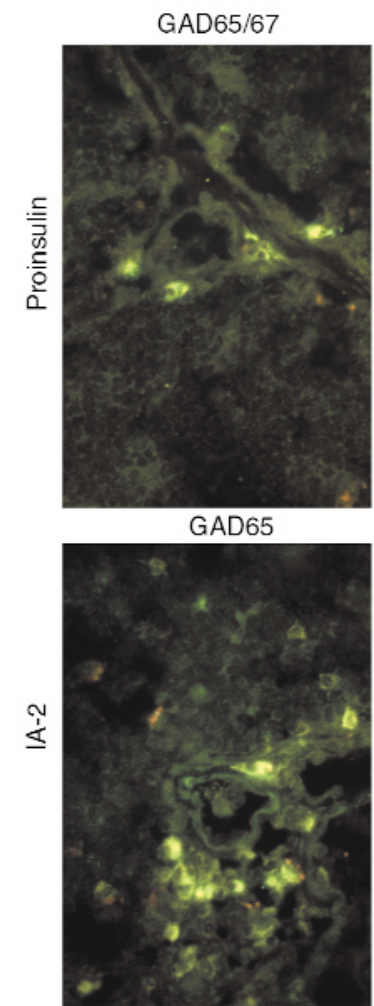

$\mathrm{IA}-2$
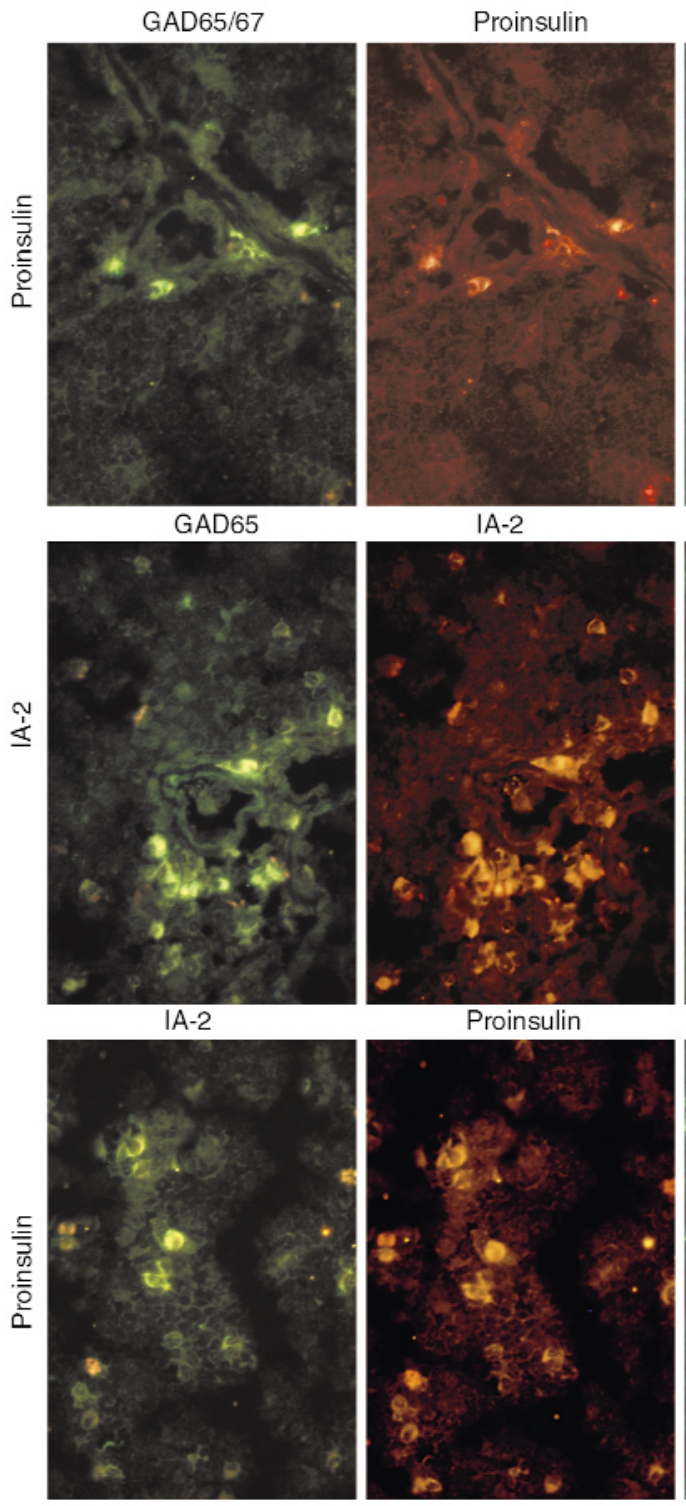

Proinsulin
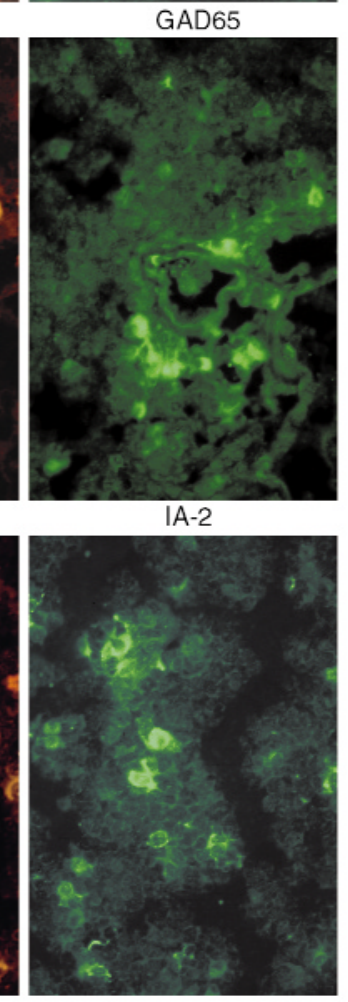

Figure 5

Self-antigen-expressing cells express multiple self-antigens. Top to bottom: thymus sections (case 1897; ×100) stained for proinsulin-GAD65/67, GAD65-IA-2, and proinsulin-IA-2. 


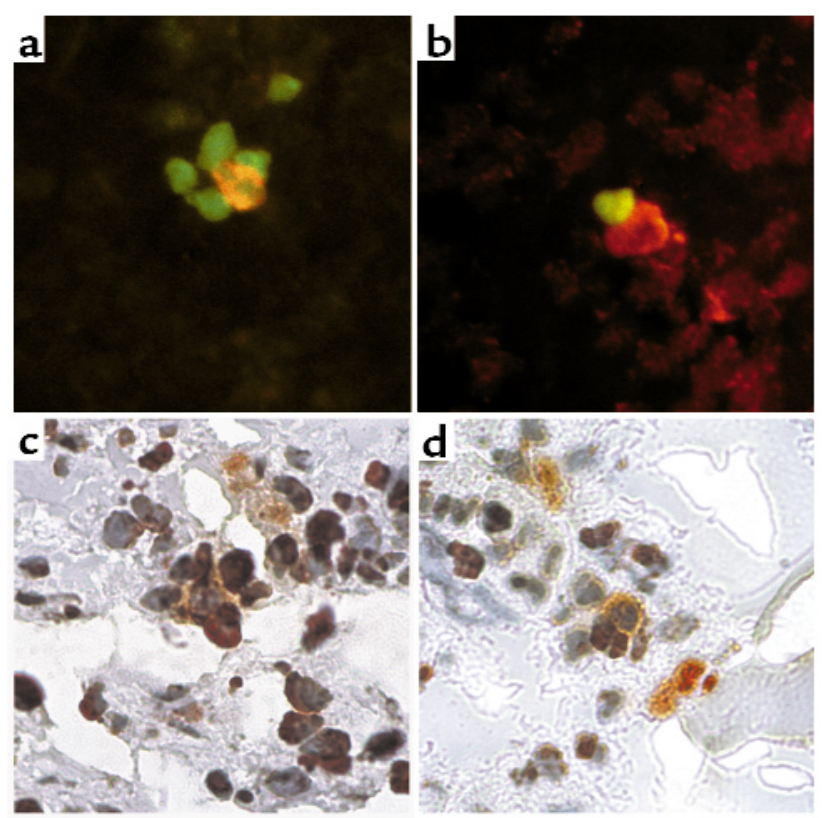

Figure 6

Self-antigen-expressing cells and apoptosis. Frozen sections were subject to TUNEL and proinsulin staining. ( $\mathbf{a}$ and $\mathbf{b}$ ) Thymus (case 1829), proinsulin (TRITC) and TUNEL (FITC) staining. $\sim \times 320$. A proinsulin-positive cell (orange fluorescence) is seen in the center of a rosette (a) surrounded by apoptotic cells (green fluorescence) or in close contact with an apoptotic cell (b). (c and d) Spleen (case 1089), proinsulin (alkaline phosphatase-AP Orange) and TUNEL staining (peroxidase-AEC). $\sim \times 320$. A large, pyramidal proinsulinpositive cell (orange) is shown in the center of a rosette surrounded by at least five cells, three of which appear TUNEL-positive (red) (c); a proinsulin-positive cell (orange) is shown in close contact with two TUNEL-positive cells (red) (d).

DCs can arise from a common myeloid progenitor, suggesting that $\mathrm{CD} 8 \alpha$ may mark differences in function and maturation status rather then ontogeny (29). Unlike mouse DCs, however, human DCs have not been shown to express CD8 $\alpha$, and thus the CD8 $\alpha$-positive cells could also be $T$ cells or cells of unknown lineage. At present, no single marker other than the selfantigens expression can distinguish DCs presenting self-antigens from stimulatory DCs. More research could define markers that individually or in combination identify tolerogenic DCs (30-32).

We found no evidence for the expression of islet cell antigens in thymic epithelial cells, as we were unable to demonstrate colocalization of the self-antigens tested with cytokeratin and AIRE. The lack of expression of AIRE also suggests that self-antigen-presenting cells differ from those DCs that express this "autoimmune regulator" molecule (24). However, our study does not exclude the possibility that thymic epithelial cells may express other peripheral self-antigens. Expression studies in sorted or otherwise purified thymic cell populations indicate that at least some peripheral self-antigens may be expressed in cells of epithelial lineage in the human thymus (17), and mouse thymic epithelial cells express proteolipid protein (33), a key autoantigen in multiple sclerosis.

The presence of self-antigen-presenting cells in lymphoid organs throughout the human life span, their association with apoptotic phenomena, and the functional evidence implicating these cells with tolerance in transgenic mice $(21,25)$ are all consistent with the notion that self-antigen expression by this subset of cells plays a key role in establishing and maintaining self-tolerance throughout life. It may appear paradoxical that IDDM or other autoimmune diseases would develop if tolerogenic cells expressing self-antigens are dispersed throughout the immune system. A plausible and currently untested hypothesis is that patients fail to express insulin and other islet cell antigens in their thymus. We could not test this hypothesis owing to the lack of tissues from patients, but this explanation seems unlikely because insulin is present in the thymus of nonobese diabetic (NOD) mice (A. Pugliese, unpublished observation; and ref. 34). It is also possible that a generic defect in antigen presentation may characterize patients with IDDM, as reduced numbers and less functionally competent DCs are reported in their peripheral blood (35). However, it is unclear whether this defect would apply to the population of DCs described here.

On the other hand, there is growing evidence that genetic polymorphisms and other regulatory mechanisms affecting self-antigen expression may influence the tolerogenic function of self-antigen-presenting cells in an antigen-specific manner. In the case of proinsulin, higher insulin mRNA levels in the thymus are associated with protection from IDDM and are genetically determined by the presence of protective variants of a polymorphic minisatellite controlling the steadystate transcription rates of the insulin gene $(15,16)$. In addition, transgenic expression of increased levels of proinsulin in the thymus (in HLA-class II-positive cells) of NOD mice is sufficient to prevent diabetes (34), and a transgenic model of myasthenia gravis shows that increased thymic levels of the acetylcholine receptor autoantigen prevents disease development (36). In contrast, parent-of-origin effects at the insulin minisatellite locus impair insulin gene expression in thymus $(15,16)$ and spleen $(37)$ by completely silencing one of the parental copies of the gene. Moreover, a mutation in the transcription factor Foxp3 causes IDDM and other autoimmune diseases in the X-linked autoimmunity-allergic disregulation syndrome, and it has been hypothesized that Foxp3 may regulate the expression of autoantigens by APCs in the thymus (38, 39). All of these observations support the notion that levels of antigen expression in the thymus play a key role in modulating thymic selection $(2,3,40-42)$. In addition, alternative splicing determines different IA-2 expression patterns in pancreas and lymphoid organs and may influence tolerance to IA-2 epitopes that are frequently targeted by autoimmune responses (43). Alternative splicing also diversifies thymus and brain 
expression of the proteolipid protein autoantigen in a model of multiple sclerosis (33). Altogether, these observations suggest that both thymic expression of peripheral proteins and qualitative and quantitative differences in their expression may significantly influence the development of self-tolerance and the risk of autoimmune disease $(15,16,21,34,36)$.

The findings reported here, assigning the expression of islet cell antigens to apparently tolerogenic self-antigen-presenting cells, provide additional support for the hypothesis just presented and yield insight into cells that may be involved in the development and maintenance of self-tolerance throughout life. Further characterization of self-antigen expression by self-antigen-presenting cells may improve our understanding of the mechanisms underlying autoimmune diseases and may help in developing specific preventive/therapeutic strategies. Some of these strategies may potentially involve self-antigen-presenting cells as an infusion of autologous tolerogenic cells. Of note, DCs have been used to prevent or modulate diabetes in NOD mice (44-46). The existence of self-antigen-expressing cells in different organs of the immune system suggests that there is potential for isolating self-antigen-presenting cells from peripheral blood for preventive and therapeutic applications. Self-antigen-expressing cells could find application not only in autoimmunity but also in transplantation, as one of the problems hampering the success of islet transplantation is the recurrence of autoimmunity against the graft $(47,48)$. Further studies to understand the role of self-antigen-presenting cells in the regulation of self-tolerance and to explore their potential for preventing and treating autoimmune disease may have clinical significance.

\section{Acknowledgments}

Tissues were provided by C.K. Petito and I. Logvisnki, University of Miami Brain and Fetal Tissue Bank (a joint effort with the University of Maryland Brain and Tissue Banks through NICHD contract NO1-HD-83284), the Diabetes Research Institute Cell Transplant Center Team, and B.F. Haynes. S. Gleason at Bayer provided rabbit antibodies to the ICA512 (IA-2) molecule. We thank T. Revann for technical assistance, R. Celestine (MicroOptics of Florida Inc.) for outstanding technical support for our microscopes, and D. Stenger for assisting in editing the manuscript. We thank S. Jayaraman for helpful discussions and critical reading of the manuscript. This work was supported by the Juvenile Diabetes Foundation International (AP, CDA 296117), and grants from the American Diabetes Association, NIH (NIAID AI-44456, AI-47604), and the Diabetes Research Institute Foundation.

1.Schwartz, R.H. 1993. Immunological tolerance. In Fundamental immunology. W.E. Paul, editor. Raven Press. New York, New York, USA. 677-731.

2. Fowlkes, B.J., and Ramsdell, F. 1993. T-cell tolerance. Curr. Opin. Immunol. 5:873-879.

3. Sprent, J., and Webb, S.R. 1995. Intrathymic and extrathymic clonal deletion of T cells. Curr. Opin. Immunol. 7:196-205.
4. Suss, G., and Shortman, K. 1996. A subclass of dendritic cells kills CD4 $T$ cells via Fas/Fas-ligand-induced apoptosis. J. Exp. Med. 183:1789-1796.

5. Fraser, A., and Evan, G. 1996. A license to kill. Cell. 85:781-784.

6. Zhang, H.G., et al. 1999. Induction of specific T cell tolerance by Fas ligand-expressing antigen-presenting cells. J. Immunol. 162:1423-1430.

7. Kyewski, B.A., Fathman, C.G., and Rouse, R.V. 1986. Intrathymic presentation of circulating non-MHC antigens by medullary dendritic cells. J. Exp. Med. 163:231-246.

8. Steinman, R.M., Pack, M., and Inaba, K. 1997. Dendritic cells in the Tcell areas of lymphoid organs. Immunol. Rev. 156:25-37.

9. Pugliese, A. 1999. Unraveling the genetics of insulin-dependent type 1A diabetes: the search must go on. Diabetes Reviews. 7:39-54.

10. Schechter, R., Beju, D., Gaffney, T., Schaefer, F., and Whetsell, L. 1996. Preproinsulin I and II mRNAs and insulin electron microscopic immunoreaction are present within the rat fetal nervous system. Brain Res. 736:16-27.

11. Devaskar, S.U., et al. 1994. Insulin gene expression and insulin synthesis in mammalian neuronal cells. J. Biol. Chem. 269:8445-8454.

12. Taha, A., Budd, G.C., and Pansky, B. 1993. Preproinsulin messenger ribonucleic acid in the rat adrenal gland. Ann. Clin. Lab. Sci. 23:469-476.

13. Budd, G.C., Pansky, B., and Glatzer, L. 1993. Preproinsulin mRNA in the rat eye. Invest. Ophthalmol. Vis. Sci. 34:463-469.

14. Mason, D., and Powrie, F. 1998. Control of immune pathology by regulatory T cells. Curr. Opin. Immunol. 10:649-655.

15. Pugliese, A., et al. 1997. The insulin gene is transcribed in the human thymus and transcription levels correlated with allelic variation at the INS VNTR-IDDM2 susceptibility locus for type 1 diabetes. Nat. Genet. 15:293-297.

16. Vafiadis, P., et al. 1997. Insulin expression in the thymus is modulated by INS VNTR alleles at the IDDM2 locus. Nat. Genet. 15:289-292.

17. Sospedra, M., et al. 1998. Transcription of a broad range of self-antigens in human thymus suggests a role for central mechanisms in tolerance toward peripheral antigens. J. Immunol. 161:5918-5929.

18. Jolicouer, C., Hanahan, D., and Smith, K.M. 1994. T-cell tolerance toward a transgenic beta-cell antigen and transcription of endogenous pancreatic genes in thymus. Proc. Natl. Acad. Sci. USA. 91:6707-6711.

19. Heath, V.L., Moore, N.C., Parnell, S.M., and Mason, D.W. 1998. Intrathymic expression of genes involved in organ specific autoimmune disease. J. Autoimmun. 11:309-318.

20. Werdelin, O., Cordes, U., and Jensen, T. 1998. Aberrant expression of tissue-specific proteins in the thymus: a hypothesis for the development of central tolerance. Scand. J. Immunol. 47:95-100.

21. Hanahan, D. 1998. Peripheral-antigen-expressing cells in thymic medulla: factors in self-tolerance and autoimmunity. Curr. Opin. Immunol. 10:656-662.

22. Solimena, M., et al. 1996. ICA 512, an autoantigen of type I diabetes, is an intrinsic membrane protein of neurosecretory granules. $E M B O \mathrm{~J}$. 15:2102-2114.

23. Jamieson, B.D., et al. 1999. Generation of functional thymocytes in the human adult. Immunity. 10:569-575.

24. Heino, M., et al. 1999. Autoimmune regulator is expressed in the cells regulating immune tolerance in thymus medulla. Biochem. Biophys. Res. Commun. 257:821-825.

25. Smith, K.M., Olson, D.C., Hirose, R., and Hanahan, D. 1997. Pancreatic gene expression in rare cells of thymic medulla: evidence for functional contribution to T cell tolerance. Int. Immunol. 9:1655-1665.

26. Throsby, M., et al. 1998. Pancreatic hormone expression in the murine thymus: localization in dendritic cells and macrophages. Endocrinology. 139:2399-2406.

27. Van Parijs, L., Ibraghimov, A., and Abbas, A.K. 1996. The roles of costimulation and Fas in T cell apoptosis and peripheral tolerance. Immunity. 4:321-328.

28. Ducoroy, P., Lesourd, M., Padros, M.R., and Tournefier, A. 1999. Natural and induced apoptosis during lymphocyte development in the axolotl. Dev. Comp. Immunol. 23:241-252.

29. Traver, D., et al. 2000. Development of CD8alpha-positive dendritic cells from a common myeloid progenitor. Science. 290:2152-2154.

30. Maldonado-Lopez, R., et al. 1999. Role of CD8alpha+ and CD8alphadendritic cells in the induction of primary immune responses in vivo. $J$. Leukoc. Biol. 66:242-246.

31. Ardavin, C. 1997. Thymic dendritic cells. Immunol. Today. 18:350-361.

32. Anjuere, F., et al. 1999. Definition of dendritic cell subpopulations present in the spleen, Peyer's patches, lymph nodes, and skin of the mouse. Blood. 93:590-598.

33. Klein, L., Klugmann, M., Nave, K.A., and Kyewski, B. 2000. Shaping of the autoreactive T-cell repertoire by a splice variant of self protein expressed in thymic epithelial cells. Nat. Med. 6:56-61.

34. French, M.B., et al. 1997. Transgenic expression of mouse proinsulin II prevents diabetes in nonobese diabetic mice. Diabetes. 46:34-39.

35. Takahashi, K., Honeyman, M.C., and Harrison, L.C. 1998. Impaired yield, phenotype, and function of monocyte-derived dendritic cells in 
humans at risk for insulin-dependent diabetes. J. Immunol. 161:2629-2635

36. Salmon, A.M., Bruand, C., Cardona, A., Changeux, J.P., and BerrihAknin, S. 1998. An acetylcholine receptor alpha subunit promoter confers intrathymic expression in transgenic mice. Implications for tolerance of a transgenic self-antigen and for autoreactivity in myasthenia gravis. J. Clin. Invest. 101:2340-2350.

37. Miceli, D., Zeller, M., Brown, D., Ricordi, C., and Pugliese, A. 1999. Insulin gene expression and imprinting in pancreas and lymphoid organs. Diabetes. 48(Suppl. 1):A190. (Abstr.)

38. Chatila, T.A., et al. 2000. JM2, encoding a fork head-related protein, is mutated in X-linked autoimmunity-allergic disregulation syndrome. J. Clin. Invest. 106:R75-R81.

39. Patel, D.D. 2001. Escape from tolerance in the human $x$-linked autoimmunity-allergic disregulation syndrome and the Scurfy mouse. J. Clin. Invest. 107:155-157.

40. Sebzda, E., et al. 1994. Positive and negative thymocyte selection induced by different concentrations of a single peptide. Science. 263:1615-1618

41. Oehen, S.U., et al. 1994. Escape of thymocytes and mature T cells from clonal deletion due to limiting tolerogen expression levels. Cell. Immunol. 158:342-352.
42. Liblau, R.S., et al. 1996. Intravenous injection of soluble antigen induces thymic and peripheral T-cell apoptosis. Proc. Natl. Acad. Sci. USA. 93:3031-3036.

43. Diez, J., et al. 2001. Differential splicing of the IA- 2 mRNA in pancreas and lymphoid organs as a permissive genetic mechanism for autoimmunity against the IA-2 IDDM autoantigen. Diabetes. In press.

44. Clare-Salzler, M.J., Brooks, J., Chai, A., Van Herle, K., and Anderson, C. 1992. Prevention of diabetes in nonobese diabetic mice by dendritic cell transfer. J. Clin. Invest. 90:741-748.

45. Feili-Hariri, M., et al. 1999. Immunotherapy of NOD mice with bone marrow-derived dendritic cells. Diabetes. 48:2300-2308.

46. Shinomiya, M., Fazle Akbar, S.M., Shinomiya, H., and Onji, M. 1999. Transfer of dendritic cells (DC) ex vivo stimulated with interferongamma (IFN-gamma) down-modulates autoimmune diabetes in nonobese diabetic (NOD) mice. Clin. Exp. Immunol. 117:38-43.

47. Jaeger, C., Brendel, M.D., Hering, B.J., Eckhard, M., and Bretzel, R.G. 1997. Progressive islet graft failure occurs significantly earlier in autoantibody-positive than in autoantibody-negative IDDM recipients of intrahepatic islet allografts. Diabetes. 46:1907-1910.

48. Roep, B.O., et al. 1999. Auto- and alloimmune reactivity to human islet allografts transplanted into type 1 diabetic patients. Diabetes. 48:484-490. 